\title{
Study of Low Frequency Phonon Modes in YBCO System
}

\author{
$\mathrm{J} \mathrm{Yu}^{1,2}, \mathrm{~L} \mathrm{Li}^{2}$, and $\mathrm{H}$ Zhang ${ }^{2, *}$ \\ ${ }^{1}$ Yellow River Conservancy Technical Institute, Kaifeng, Henan, 475004, China; yujing@yrcti.edu.cn \\ ${ }^{2}$ Materials Physics Laboratory, State Key Laboratory for Mesoscopic Physics, Department of Physics, Peking \\ University, Beijing 100871, China \\ * hanzhang@pku.edu.cn; +86 (010)6275 4233
}

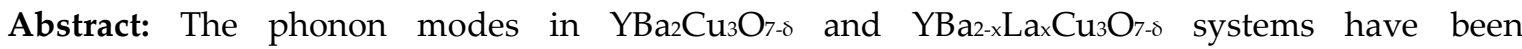
systematically studied by Raman spectroscopy. The new phonon modes of $104 \mathrm{~cm}^{-1}, 94 \mathrm{~cm}^{-1}$, and 89 $\mathrm{cm}^{-1}$ were found in all these samples. A crude estimate about the wavenumber of the collective vibration of the stable $\mathrm{CuO} 2$ plane was given in this paper. The standard deviations of the new phonons in $\mathrm{YBa}_{2} \mathrm{Cu}_{3} \mathrm{O}_{7-\delta}$ and $\mathrm{YBa}_{2}-\mathrm{x} \mathrm{La}_{x} \mathrm{Cu}_{3} \mathrm{O}_{7-\delta}$ systems were discussed. The results of the calculation indicated that the $104 \mathrm{~cm}^{-1}$ mode probably stands the c-direction collective vibration of the stable $\mathrm{CuO} 2$ plane, the $94 \mathrm{~cm}^{-1}$ mode stands the a-direction vibration, and the $89 \mathrm{~cm}^{-1}$ mode stands b-direction vibration. The relevance between these phonons and the superconductivity was discussed. It is found that, as the $T_{c}$ decreased, the $104 \mathrm{~cm}^{-1}$ mode and the $94 \mathrm{~cm}^{-1}$ mode softened, and the $89 \mathrm{~cm}^{-1}$ mode hardened slightly.
\end{abstract}

Keywords: collective vibration; raman spectroscopy; superconductivity; $\mathrm{CuO} 2$ plane; $\mathrm{YBCO}$ system

*Yu and Li equally contributed to this paper.

\section{Introduction}

High temperature superconductor (HTSC) has been found for many years, but its mechanism is still open. Conventional BCS theory [1], which core is electron-phonon interaction (EPI), once was abandoned by most researchers as some important properties of HTSC cannot been explained well by it. At the same time, many theories appeared to describe the HTSC, such as resonating valence bond (RVB) theory [2], exciton model [3], strong coupling theory [4], and so on. However, all these new theories were inadequate to account for the mechanism of HTSC. In 2001, Lanzara [5] et al studied different families of the cuprates with angle-resolved photoemission spectroscopy (ARPES), and they observed an abrupt change of the electron velocity at 50-80 meV. Meevasana [6] ascribed the "kink" to the coupling between electrons and special phonons of some collective behavior. Subsequently, many experiments focused on EPI occurred. Khasanov [7] observed evident oxygen-isotope $(16 \mathrm{O} / 18 \mathrm{O})$ effect directly in the in-plane penetration depth of YBCO film, which demonstrated the significance of the EPI in the cuprates. Venturini et al [8] observed a pair-breaking peak in electron Raman spectra of Bi2212 when the samples were cooled below the critical temperature. Pallesy [9] et al reported that the frequencies of some phonons would change gradually with the content of $\mathrm{Ca}$ in $\mathrm{Y}_{1-\mathrm{x}} \mathrm{Ca}_{\times} \mathrm{Ba}_{2} \mathrm{Cu}_{3} \mathrm{O}_{7-\delta}$ system. And Jin et al [10] obtained similar results in $\mathrm{YBa}_{2}-\mathrm{xLa}_{2} \mathrm{Cu}_{3} \mathrm{O}_{7-\delta}$ system. In our previous work, these phenomenon was confirmed in $\mathrm{Y}_{1-\mathrm{X}} \mathrm{Ca}_{\times} \mathrm{Ba}_{2-\mathrm{x}} \mathrm{La}_{\mathrm{C}} \mathrm{Cu}_{3} \mathrm{O}_{7-\delta}$ system [11]. All these experiments demonstrated the dominance of phonons in 
HTSC. Some review articles emphasized the importance of the EPI in the cuprates also [12, 13]. Recently, some researchers [14] discovered pressurised hydrogen sulphide with $T_{c}$ exceeding 200 $\mathrm{K}$, which is a conventional superconductor. This result means that there is no limitation of Tc value for conventional superconductors, and EPI can result in very high Tc value superconductors.

Coincidently, some experiments proved that the stable $\mathrm{CuO} 2$ plane plays a great role in HTSC [10, 15-17]. Some researchers pointed out that there may exist a collective vibration of the stable $\mathrm{CuO} 2$ plane, which probably is the core of the EPI in HTSC. Guo et al [15] reported that they had calculated out the wavenumber of the collective vibration in c-direction of the stable $\mathrm{CuO} 2$ plane in $\mathrm{YBa}_{2} \mathrm{Cu}_{3} \mathrm{O}_{7-\delta}$ system as about $105 \mathrm{~cm}^{-1}$. However, phonons of the collective vibration have not been accurately observed yet and the relationship between the phonon and superconductivity is still open. A further study on the collective vibration of the stable $\mathrm{CuO} 2$ plane in cuprates is helpful to make clear whether the EPI is significant to superconductivity or not. In this paper, $\mathrm{YBa}_{2} \mathrm{Cu}_{3} \mathrm{O}_{7-\delta}$

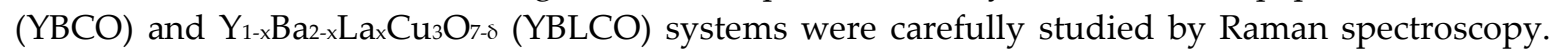
Fortunately, we found three new modes of phonons, which acted like the collective vibration of the stable $\mathrm{CuO} 2$ plane. By analyzation, the $104 \mathrm{~cm}^{-1}$ mode probably stands the c-direction collective vibration of the stable $\mathrm{CuO} 2$ plane, the $94 \mathrm{~cm}^{-1}$ mode stands the a-direction vibration, and the $89 \mathrm{~cm}^{-1}$ mode stands b-direction vibration. The relationship between the phonons and the superconductivity shows a positive evidence for EPI in HTSC.

\section{Materials and Methods}

The samples of $\mathrm{YBa}_{2} \mathrm{Cu}_{3} \mathrm{O}_{7-\delta}(\delta=0.08,0.19,0.23,0.32,0.51), \mathrm{YBa}_{-\mathrm{x}} \mathrm{La}_{\mathrm{x}} \mathrm{Cu}_{3} \mathrm{O}_{7-\delta}(\delta=0.08, \mathrm{x}=0,0.1,0.2$, $0.3,0.4)$ were prepared by the standard solid-state reaction method. An X'pert MRD diffractometer with $\mathrm{Cu}^{\mathrm{Ku}}$ radiation was used to collect $\mathrm{X}$-ray powder diffraction data. Rietveld refinement method with the X'pert Plus software was used to get the lattice parameters. The Tc was determined in a 20-Oe magnetic field by dc magnetic susceptibility measurements (Quantum Design MPMS). The samples of $\mathrm{YBa}_{2} \mathrm{Cu}_{3} \mathrm{O}_{7-\delta}(\delta=0.19,0.23,0.32,0.51)$ was obtained from $\mathrm{YBa}_{2} \mathrm{Cu}_{3} \mathrm{O}_{7-\delta}(\delta=0.08)$ by releasing $\mathrm{O}$ atom in $\mathrm{Ar}$ atmosphere for different time. The oxygen contents were got by a method, estimating the oxygen content by the value of $T_{c}[18,19]$.

All the Raman spectra were collected from $50 \mathrm{~cm}^{-1}$ to $800 \mathrm{~cm}^{-1}$ at room temperature with a SP-2500 spectrometer equipped with a microscope and a CCD detector. The wavelength of laser is $532 \mathrm{~nm}$. And the laser was focused to a spot of $2 \mu \mathrm{m}$ in diameter.

\section{Results}

In the $\mathrm{YBCO}$ system, the Roman spectra are shown in Figure 1. It is found that there were new peaks around $100 \mathrm{~cm}^{-1}$ in every sample never reported, which indicates new modes of phonons. For a further study, we picked the period nearby $100 \mathrm{~cm}^{-1}$ of the spectra and fitted these curves by Lorentz shapes, respectively. The adjusted $\mathrm{R}$ squares (Adj. R-Squares) of the fittings are all lager than 0.99 , which demonstrates that the results were convincible. The peaks was consisted of $104 \mathrm{~cm}^{-1}$, $94 \mathrm{~cm}^{-1}$, and $89 \mathrm{~cm}^{-1}$ modes of phonons, which were shown in Figure 2. 


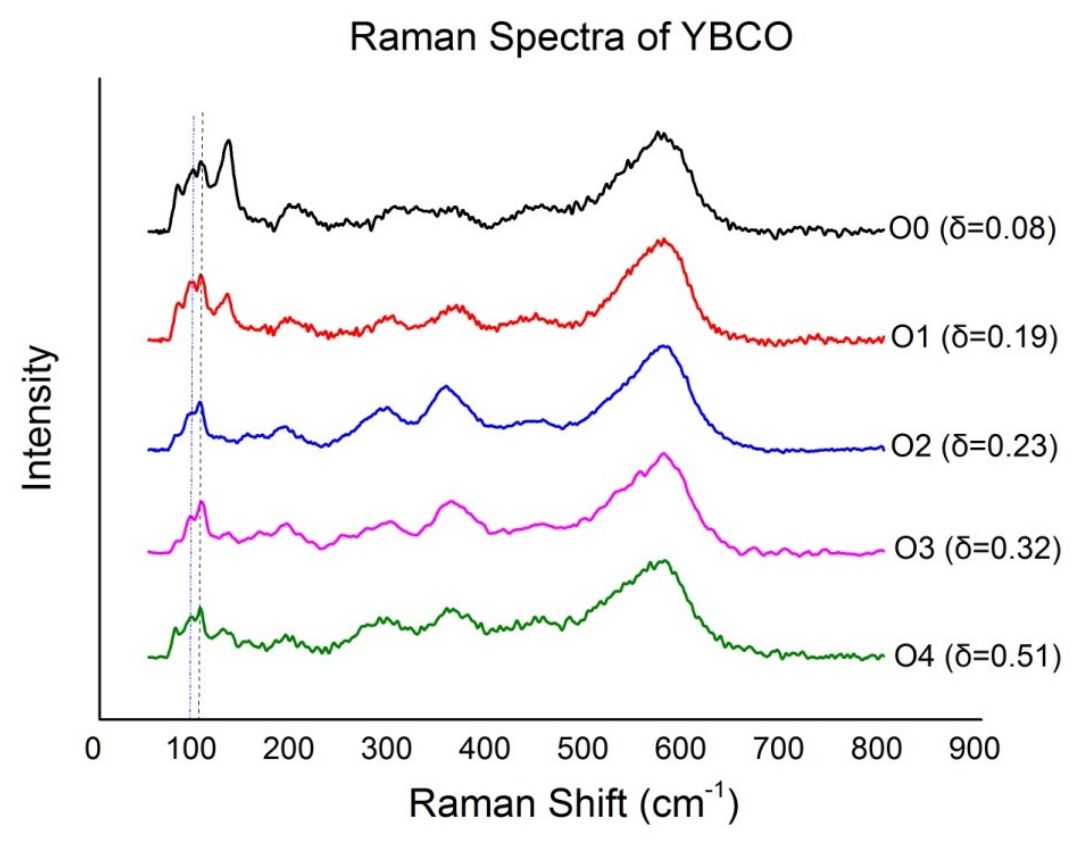

Fig. 1. Raman Spectra of theYBCO system. New peaks around $100 \mathrm{~cm}^{-1}$ were found.

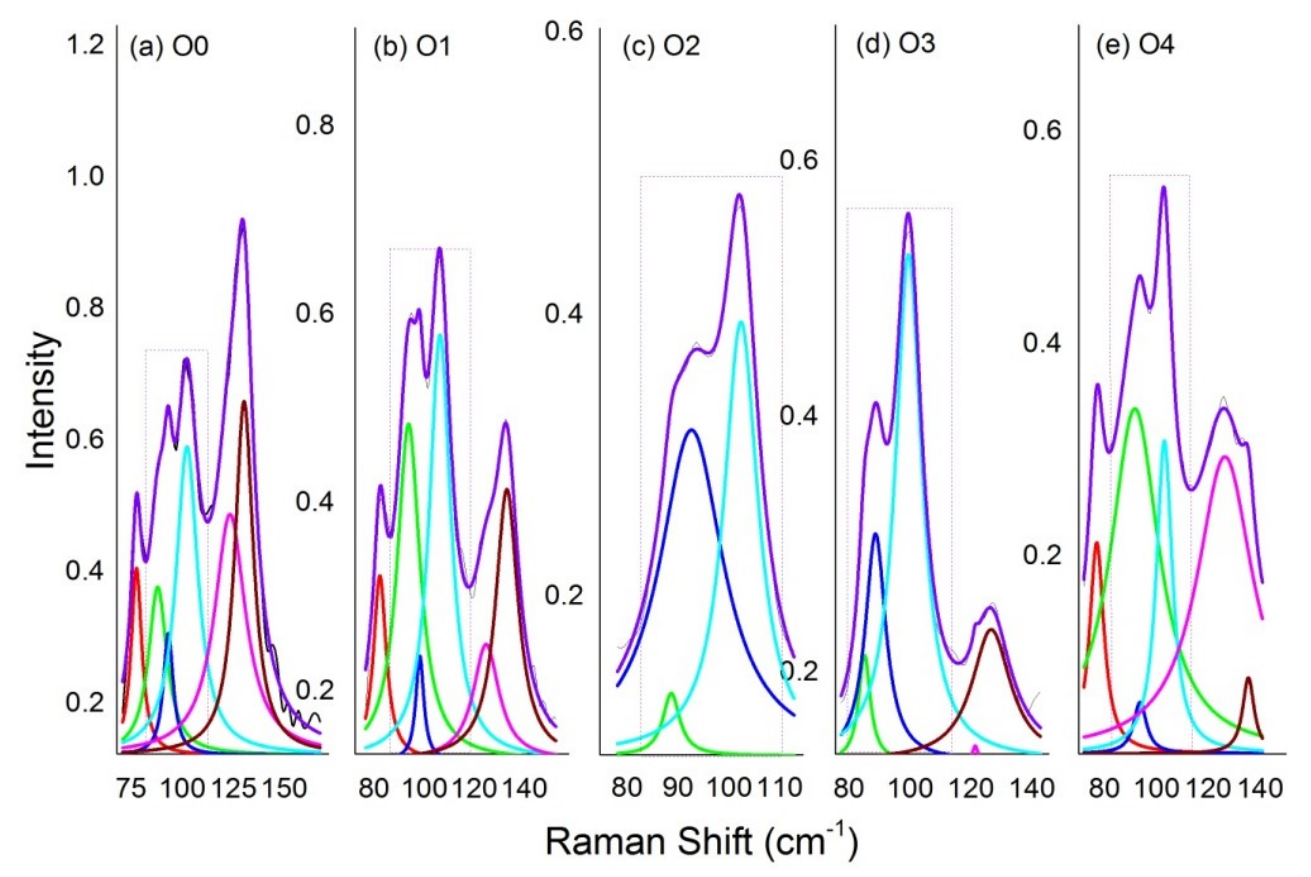

Fig. 2. Fitted Lorentz shape in YBCO system. The Adj. R-Square were all large than 0.99, indicating that the fitting results were reliable.

In the YBLCO system, the Raman spectra are shown in Figure 3. Also there existed new peaks around $100 \mathrm{~cm}^{-1}$ in each sample. Also the period nearby the $100 \mathrm{~cm}^{-1}$ mode were picked and fitted with Lorentz shape. The results were shown in Figure 4. The $104 \mathrm{~cm}^{-1}, 94 \mathrm{~cm}^{-1}$, and $89 \mathrm{~cm}^{-1}$ modes existed in YBLCO system too. 


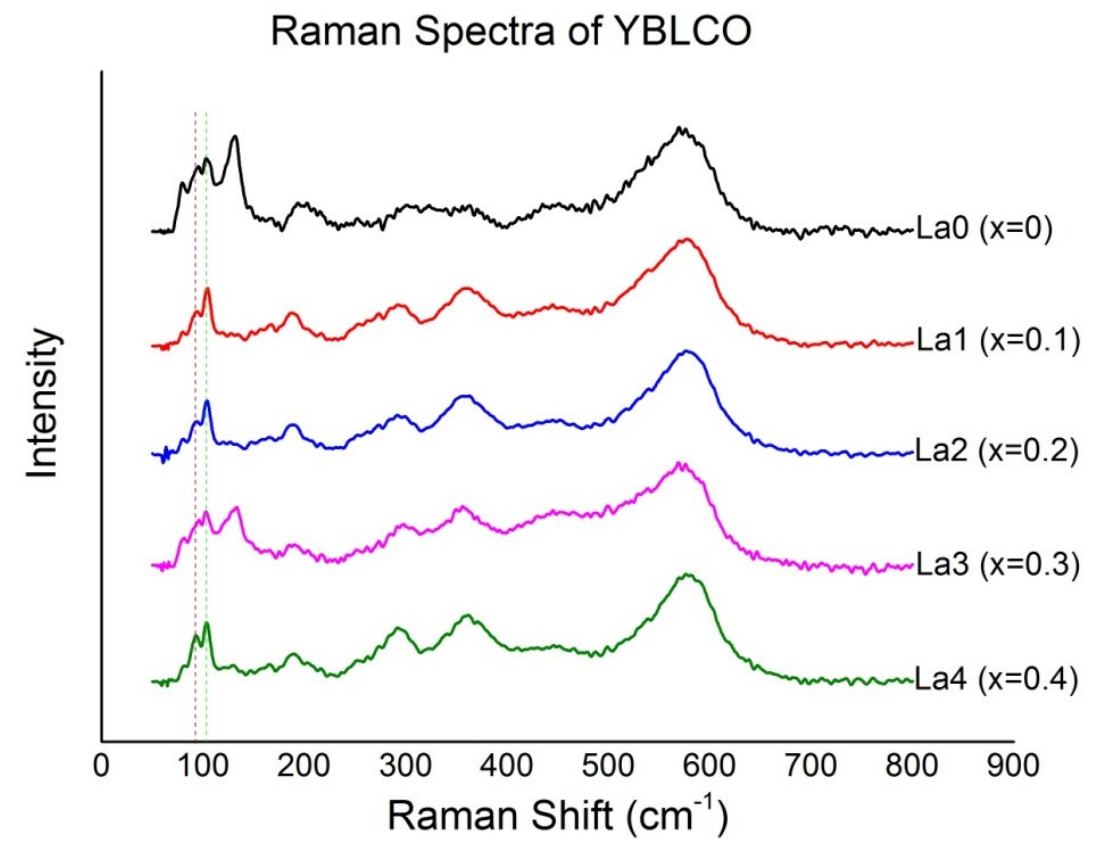

Fig. 3. Raman spectra of YBLCO system. New peaks around $100 \mathrm{~cm}^{-1}$ were found.

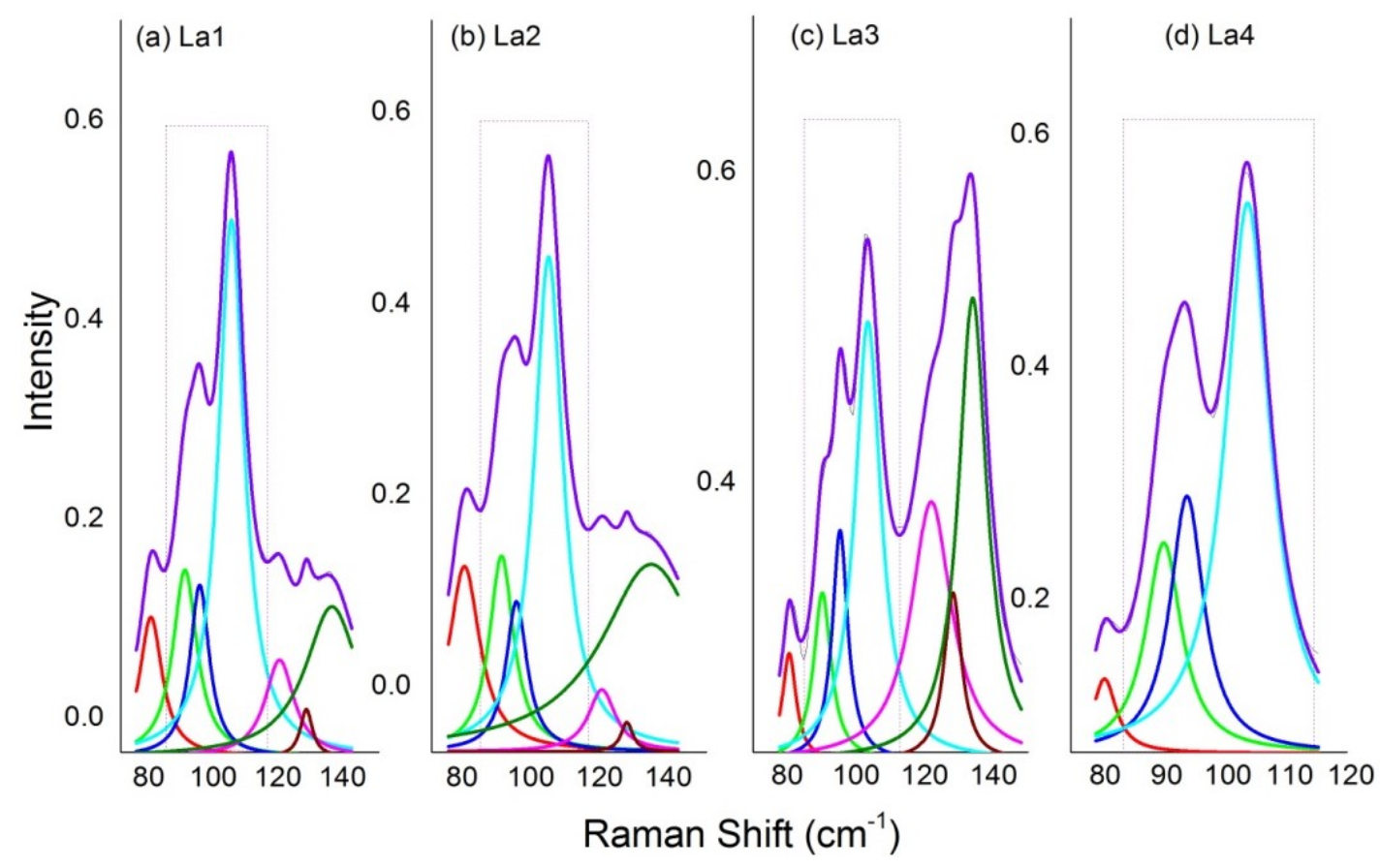

Fig. 4. Fitted Lorentz shape in YBLCO system. The Adj. R-Square were all large than 0.99, indicating that the fitting results were reliable.

\section{Discussion}

These three new phonons had never been reported. Maybe collective vibration of the stable $\mathrm{CuO} 2$ plane should be taken into consideration. Some authors [10, 15-17, 20, 21] have demonstrated by $\mathrm{x}$-ray diffraction and Raman spectroscopy that $\mathrm{CuO} 2$ plane is very stable, and the bond length and bond angle in this plane is hardly changed by the variations of environments. The atoms in this plane may show some behaviors of collective movements, and they may play an important role in high Tc superconductivity. However, about these collective movements and their role in high Tc superconductivity is not clear. Here we try to understand it by analyzing of the Raman spectra got in this study. Guo et al [15] reported the calculation about the collective vibration in YBCO sample. 
They assumed that the frequencies of phonons are inverse-square to its weight, the cluster of the stable $\mathrm{CuO} 2$ plane vibrates like a single atom, and the stable $\mathrm{CuO} 2$ plane is consisted of one $\mathrm{Cu}$ atom and four $\mathrm{O}$ atoms and they deduced the frequency of the phonon of the c-direction vibration of the stable $\mathrm{CuO} 2$ plane was about $106 \mathrm{~cm}^{-1}$. In our model, the assumption that the frequencies of phonons are inverse-square to its weight and the cluster of the stable $\mathrm{CuO} 2$ plane vibrates like a single atom was accepted. However, what was taken into consideration is that the $\mathrm{Cu}$ and $\mathrm{O}$ atoms was not just belong to the given unit cell, but shared with the others. Figure 5 shows the unit cell of YBCO. The $\mathrm{Cu}(2)$ atom was shared by four cells, and the $\mathrm{O}(2)$ and $\mathrm{O}(3)$ atoms were shared by two cells. In our model, only a quarter of $\mathrm{Cu}(2)$ atom, half $\mathrm{O}(2)$ atom and half $\mathrm{O}(3)$ atom were taken into consideration as shown in Figure 6.

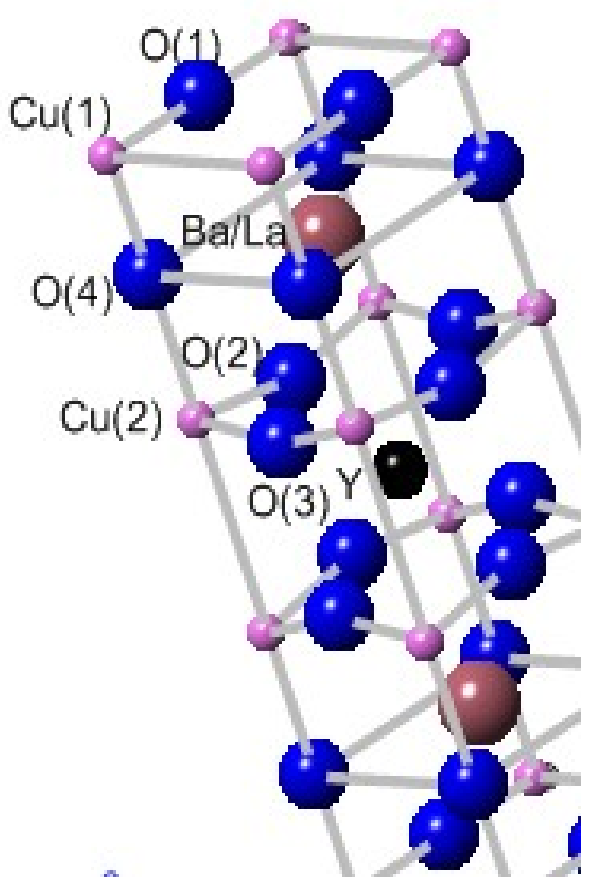

Fig. 5. Cell structure of YBCO.

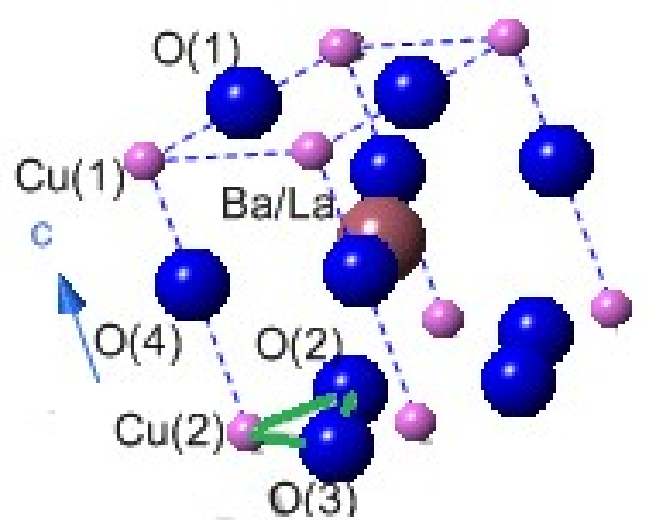

Fig. 6. The c-direction vibration of the stable $\mathrm{CuO} 2$ plane.

Thus, the ratio of the frequencies was deduced as 


$$
\frac{\omega_{\mathrm{c}}}{\omega_{\mathrm{Cu}}}=\sqrt{\frac{\frac{1}{4} \mathrm{~m}_{\mathrm{Cu}}}{\frac{1}{2} m_{o}+\frac{1}{2} m_{o}+\frac{1}{4} m_{C u}} \approx 0.707}
$$

The frequency of the c-direction vibration of $\mathrm{Cu}(2)$ was reported as about $145 \mathrm{~cm}^{-1}$ [22]. The c-direction vibration of the stable $\mathrm{CuO} 2$ plane could be obtained as $\omega_{c} \approx 145 \mathrm{~cm}^{-1} \times 0.707 \approx 102.5 \mathrm{~cm}^{-1}$. This result was coincident with the $104 \mathrm{~cm}^{-1}$ mode obtained above. However, no explanations have been given for $94 \mathrm{~cm}^{-1}$ and $89 \mathrm{~cm}^{-1}$ modes of phonons.

In our model, we took the a-direction and b-direction of the stable $\mathrm{CuO} 2$ plane into consideration also. We assumed that the nearest $\mathrm{O}$ atoms would vibrate together with the stable $\mathrm{CuO} 2$ plane as a single $\mathrm{O}$ atom. In c-direction, as shown in Fig. 5, the $\mathrm{Cu}(1)$ atom of heavy mass stand in the way of the c-direction vibration of $\mathrm{O}(4)$ atom. Thus the $\mathrm{O}(4)$ atom, the nearest atom to the stable $\mathrm{CuO} 2$ plane cannot vibrate together in c-direction. But in a-direction, as there is no roadblock, the $\mathrm{O}(4)$ atom, should vibrate together with the stable $\mathrm{CuO} 2$ plane. In this condition, as shown in Figure 7, a triangular pyramid was formed by $\mathrm{Cu}(2), \mathrm{O}(2), \mathrm{O}(3)$, and $\mathrm{O}(4)$ atoms, vibrating together.

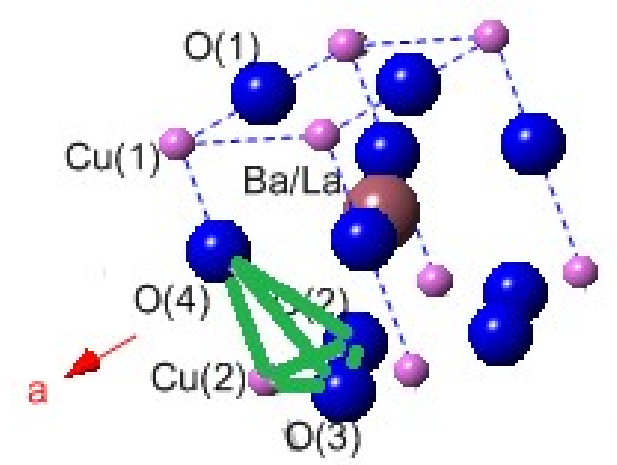

Fig. 7. The a-direction vibration of the stable $\mathrm{CuO} 2$ plane. The $\mathrm{O}(4)$ atom vibrates together with the stable $\mathrm{CuO} 2$ plane.

Noticed that the $\mathrm{O}(4)$ atom was shared by four cells, the ratio of the frequencies was deduced as

$$
\frac{\omega_{o}}{\omega_{C u}}=\sqrt{\frac{\frac{1}{4} m_{C u}}{\frac{1}{2} m_{o}+\frac{1}{2} m_{o}+\frac{1}{4} m_{o}+\frac{1}{4} m_{C u}}} \approx 0.667
$$

The frequency of the a-direction or b-direction of the $\mathrm{Cu}(2)$ was reported about $140 \mathrm{~cm}^{-1}$ [22]. We work out that phonon in the a-direction is $\omega_{a} \approx 140 \mathrm{~cm}^{-1} \times 0.667=93.4 \mathrm{~cm}^{-1}$, which matches very well with the new mode of $94 \mathrm{~cm}^{-1}$.

In the b-direction, we assumed that the $\mathrm{O}(1)$ atom, as the nearest $\mathrm{O}$ atom to the triangular pyramid, contributes to the phonon also. In the a-direction, the $\mathrm{O}(1)$ atom cannot move together with the stable $\mathrm{CuO} 2$ plane, as the $\mathrm{Cu}(1)$ atom stand in the way of the a-direction vibration of the $\mathrm{O}(1)$ atom. But in b-direction, there was no roadblock in the b-direction vibration of the $\mathrm{O}(1)$ atom. Thus, we assumed that the $\mathrm{Cu}(2)$ atom and the $\mathrm{O}$ atoms vibrated together, as shown in Figure 8. 


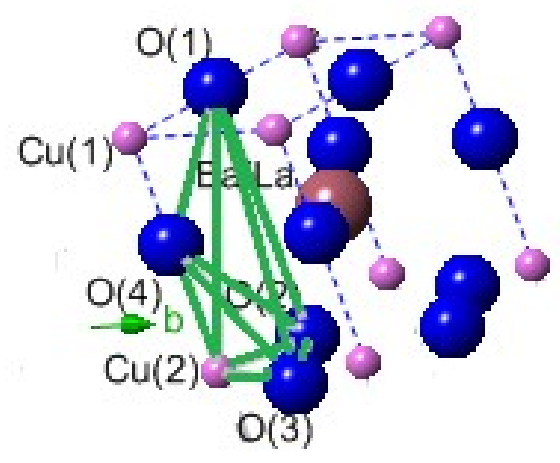

Fig. 8. The b-direction vibration of the stable $\mathrm{CuO} 2$ plane. The $\mathrm{O}(4)$ atom and the $\mathrm{O}(1)$ atom vibrate together with the stable $\mathrm{CuO} 2$ plane.

Considering that the $\mathrm{O}(1)$ atom was shared by four cells also, the ratio of the frequencies was deduced as

$$
\frac{\omega_{b}}{\omega_{C u}}=\sqrt{\frac{\frac{1}{4} m_{C u}}{\frac{1}{2} m_{o}+\frac{1}{2} m_{o}+\frac{1}{4} m_{o}+\frac{1}{4} m_{o}+\frac{1}{4} m_{C u}}} \approx 0.632
$$

We work out the phonon in the b-direction is $\omega_{b} \approx 140 \mathrm{~cm}^{-1} \times 0.632=88.5 \mathrm{~cm}^{-1}$, which matches very well with the new mode of $89 \mathrm{~cm}^{-1}$.

Another evidence to support our opinion is that the fluctuations of the frequencies of these three modes are not as drastic as others. In YBCO system, Figure 9(a) compared these modes with the $580 \mathrm{~cm}^{-1}$ mode, which represents the b-direction vibration of the $\mathrm{O}(2)$ atom [22] and was obtained by fitted Lorentz shape also. And Figure 9(b) shows their standard deviation (STDEV). It is found that the STDEV of $580 \mathrm{~cm}^{-1}$ mode is about two times as large as the $94 \mathrm{~cm}^{-1}$ mode or $89 \mathrm{~cm}^{-1}$ mode, and three times as large as the $104 \mathrm{~cm}^{-1}$ mode. As reported that the $\mathrm{CuO} 2$ plane in HTSCS is very stable [16], and the environment change hardly effects its structure. The standard deviations of the phonons related with the stable $\mathrm{CuO} 2$ plane should be very small. Coincidently, the similar phenomenon was observed in YBLCO system. The results are shown in Figure 10.

\section{YBCO System}
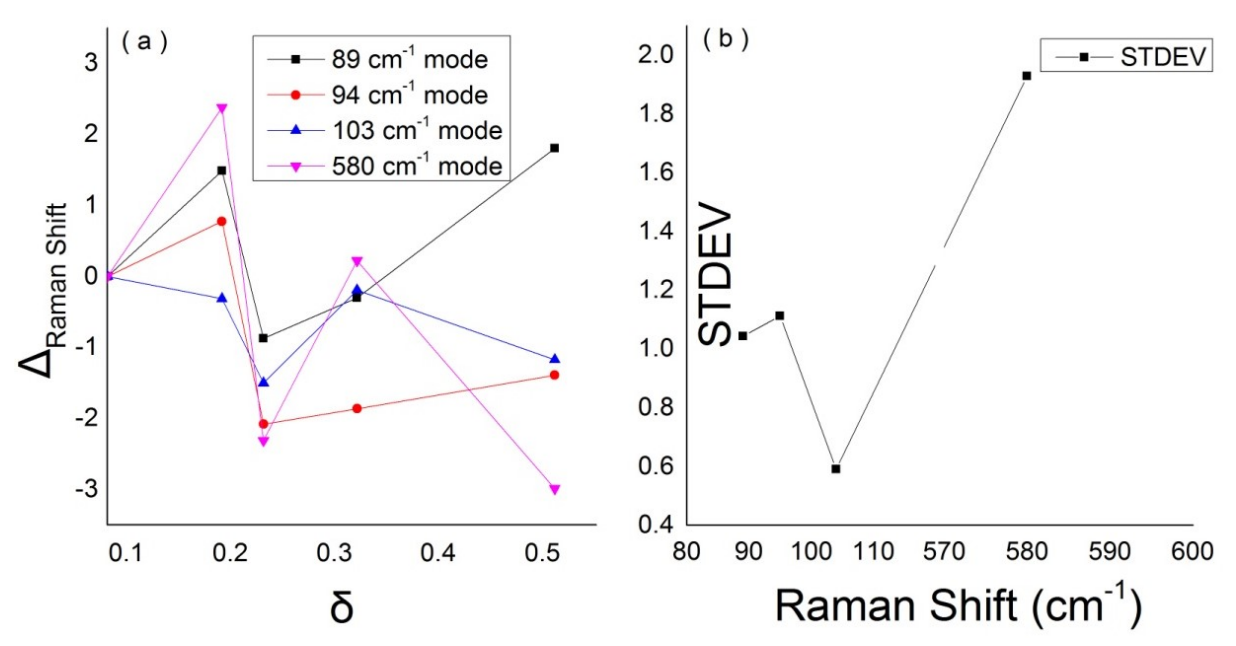

Fig. 9. (a) Fluctuations of the phonon modes in YBCO system; (b) STDEV of the phonons of in YBCO system. 


\section{YBLCO System}
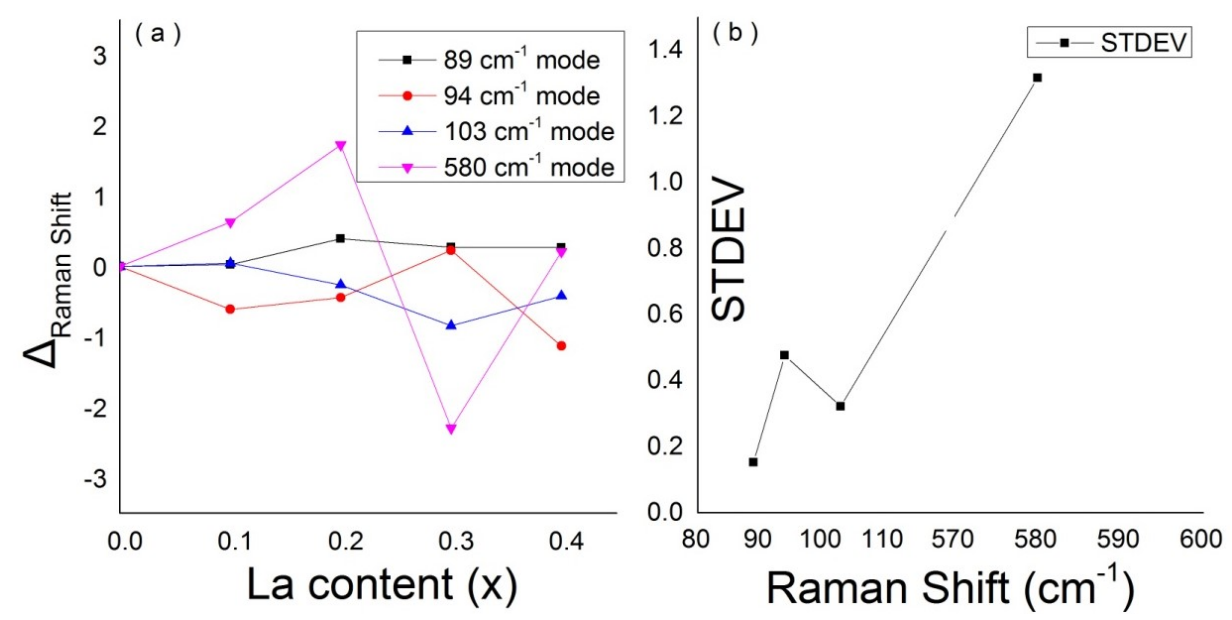

Fig. 10. (a) Fluctuations of the phonon modes in YBLCO system; (b) STDEV of the phonons of in YBLCO system.

Further searching about the relationship between the change of phonons and the superconductivity, we depicted the fluctuations of the phonon wavenumbers and the Tc in Figure 11. The wavenumbers were deposed by linear fitting. It is found that, in YBCO system, the $89 \mathrm{~cm}^{-1}$ mode phonon hardened slightly as the Tc decreased. On the contrary, the $94 \mathrm{~cm}^{-1}$ mode phonon and $104 \mathrm{~cm}^{-1}$ mode phonon softened obviously with the decreases of the Tc. The same trend was observed in YBLCO system. It indicated that the collective vibration of the stable $\mathrm{CuO} 2$ plane plays a great role in HTSC.

\section{Relevance between Phonons and $T_{c}$}
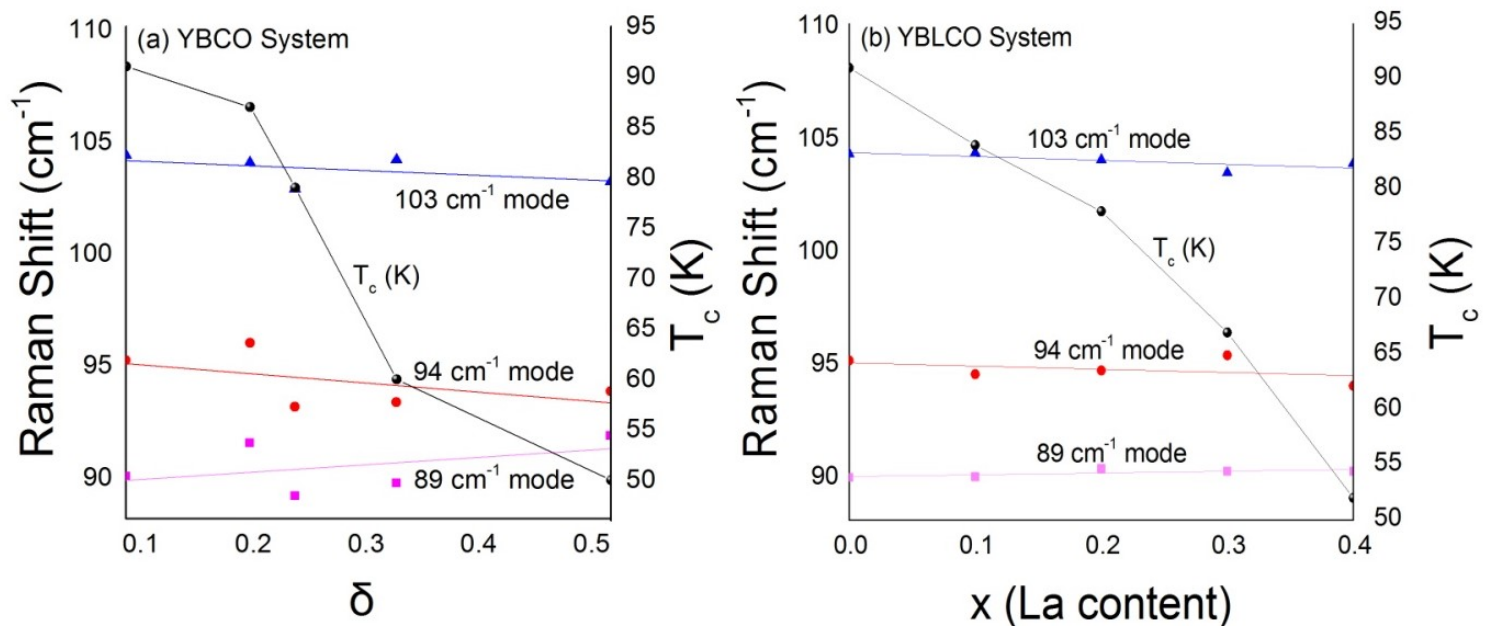

Fig.11. Relevance between the phonons and superconductivity. In both YBCO and YBLCO system, as the Tc decreased, the $89 \mathrm{~cm}^{-1}$ mode phonon hardened, but the $104 \mathrm{~cm}^{-1}$ mode and the $94 \mathrm{~cm}^{-1}$ mode phonons softened.

\section{Conclusions}

The low frequency phonon modes in YBCO and YBLCO system were studied by Raman spectroscopy. Three new modes of phonon around $100 \mathrm{~cm}^{-1}$ were found and a possible explanation relevant with collective behavior of the $\mathrm{CuO} 2$ plane was given. By computation, we found that the 
$104 \mathrm{~cm}^{-1}$ mode probably represents the c-direction collective vibration of the stable $\mathrm{CuO} 2$ plane, the $94 \mathrm{~cm}^{-1}$ mode represents the a-direction vibration, and the $89 \mathrm{~cm}^{-1}$ mode represents the b-direction vibration of the $\mathrm{CuO} 2$ plane. Besides, the relevance between the collective vibration and superconductivity is revealed. In both YBCO and YBLCO system, as the $T_{c}$ decreased, the $104 \mathrm{~cm}^{-1}$ mode and $94 \mathrm{~cm}^{-1}$ mode phonons softened, and the $89 \mathrm{~cm}^{-1}$ mode phonon hardened. The results hint that the phonons of the collective vibration of the stable $\mathrm{CuO} 2$ plane obviously contribute to the high $T_{c}$ superconductivity and need to be further studied.

\section{Acknowledgments}

The authors are grateful for the support of the State Key Laboratory for Mesoscopic Physics at Peking University.

\section{References}

1. Bardeen J, Cooper L N; Schrieffer J R. Theory of Superconductivity. Phys. Rev. 1957, 108, 1175, DOI: 10.1103/PhysRev.108.1175.

2. Anderson P W. The Resonating Valence Bond State in La2CuO4 and Superconductivity. Science 1987, 235, 1196, DOI: 10.1126/science.235.4793.1196.

3. Hayden S M; Mook H A; Dai P C; Perring T C; Dogan F. The structure of the high-energy spin excitations in a high-transition-temperature superconductor. Nature 2004, 429, 531, DOI: 10.1038/nature02576.

4. Zeyher R; Zwicknagl G. Superconductivity-induced phonon self-energy effects in high- $\mathrm{T}_{\mathrm{c}}$ superconductors. Z. Phys. B 1990, 78, 175, DOI: 10.1007/BF01307833.

5. Lanzara A; Bogdanov P V; Zhou X J; Kellar S A; Feng D L; Lu E D; Yoshida T; Eisaki H; Fujimori A; Kishio K, Shimoyama J-I; Nodak T; Uchidak S; Hussain Z; and Shen Z X. Evidence for ubiquitous strong electron-phonon coupling in high-temperature superconductors. Nature 2001, 412, 510, DOI:10.1038/35087518.

6. $\quad$ Meevasana W; Ingle N J; Lu D H; Shi J R; Baumberger F; Shen K M; Lee W S; Cuk T; Eisaki H; Devereaux T P; Nagaosa N; Zaanen J; and Shen Z X. Doping dependence of the coupling of electrons to bosonic modes in the single-layer high-temperature $\mathrm{Bi}_{2} \mathrm{Sr}_{2} \mathrm{CuO}_{6}$ superconductor. Phys. Rev. Lett. 2006, 96, 157003, DOI: 10.1103/PhysRevLett.96.157003.

7. Khasanov R; Eshchenko D G; Luetkens H; Morenzoni E; Prokscha T; Suter A; Garifianov N; Mali M; Roos J; Conder K; and Keller H. Direct Observation of the Oxygen Isotope Effect on the In-Plane Magnetic Field Penetration Depth in Optimally Doped $\mathrm{YBa}_{2} \mathrm{Cu}_{3} \mathrm{O}_{7-\delta}$. Phys. Rev. Lett. 2004, 92, 057602, DOI:10.1103/PhysRevLett.92.057602.

8. Venturini F; Opel M; Devereaux T P; Freericks J K; Tüttő I; Revaz B; Walker E; Berger H; Forró L; Hackl R. Observation of an Unconventional Metal-Insulator Transition in Over doped CuO2 Compounds. Phys. Rev. Lett. 2002, 89, 107003, DOI:10.1103/PhysRevLett.89.107003.

9. Pallesy D; Liarokapis E; Leventouri Th; Chakoumakos B C. The effect of Ca substitution on the structure

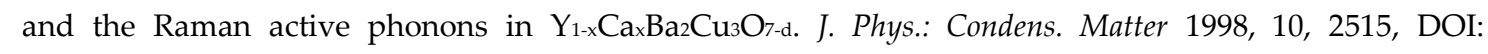
10.1088/0953-8984/10/11/015.

10. Jin W T, Hao S J; Wang C X; Guo C Q; Xia L; Zhang S L; Narlikar A V; Zhang H. Structural and spectroscopic evidence for stable chemical bonds and the correlation with high Tc superconductivity. Supercond. Sci. Technol. 2012, 25, 065004, DOI: 10.1088/0953-2048/25/6/065004.

11. Li L; Guo C Q; Han J X; Yan Y; Jin W T; Hao S J; Lin F; Wei K; Zang H. Role of lattice in YBCO superconductors studied by Raman spectroscopy. International Journal of Modern Physics 2015, 29, 5, DOI: 10.1142/S0217979215420047.

12. Kresin V Z; Wolf S A. Electron-lattice interaction and its impact on high Tc superconductivity. Rev. Mod. Phys. 2009,81, 481, DOI:10.1103/RevModPhys.81.481.

13. Johnston S; Lee W S; Chen Y; Nowadnick E A; Moritz B; Shen Z-X; Devereaux T P. Material and Doping Dependence of the Nodal and Antinodal Dispersion Renormalizations in Single- and Multilayer Cuprates. Adv. Condens. Matter. Phys. 2010, 2010, 968304, DOI: http:10.1155/2010/968304.

14. Drozdov A P; Eremets M I; Troyan I A; Ksenofontov V; Shylin S I. Conventional superconductivity at 203 kelvin at high pressures in the sulfur hydride system. Nature 2015, 525, 73, DOI: 10.1038/nature14964. 
15. Guo C Q; Yu J; Hao S J; Jin W T; Guo W; Zhang H. Low frequency phonon model in YxPr1-xBa2Cu3O7 system. Physic C 2013, 493, 60, DOI: j.physc.2013.03.025.

16. Hao S J; Jin W T; Guo C Q; Zhang $\mathrm{H}$. The stability of the $\mathrm{CuO} 2$ plane and its influence on superconductivity in the doped $\mathrm{YBa}_{2} \mathrm{Cu}_{3} \mathrm{O}_{7-\delta}$ system. Supercond. Sci. Technol. 2013, 26, 065011, DOI:10.1088/0953-2048/26/6/065011.

17. L. Li; C. Q. Guo; J. X. Han; Y. Yan; W. T. Jin. Role of lattice in YBCO superconductors studied by Raman spectroscopy. International Journal of Modern Physics 2015, 29, 1542004, DOI: 10.1142/S0217979215420047.

18. Jorgensen J D; Veal B; W, Paulikas A P; Nowicki L J; Crabtree G W; Clause H; Kwok W K. Structural properties of oxygen-deficient $\mathrm{YBa}_{2} \mathrm{Cu}_{3} \mathrm{O}_{7-\delta}$. Phys. Rev. B 1990, 41, 1863, DOI:10.1103/PhysRevB.41.1863.

19. Cava R J; Batlogg B; Fleming R M; Sunshine S A; Ramirez A; Rietman E A; Zahurak S M; an Dover R B. $\mathrm{Ba}_{2-x \mathrm{La}} \mathrm{YCu}_{3} \mathrm{O}_{7 \pm \delta}$ perovskite compounds: Crystal chemistry. Phys. Rev. B 1988, 37, 5912, DOI:10.1103/PhysRevB.37.5912.

20. Jin W T; Hao S J; Zhang H. The fixed triangle chemical bond and its effect in the Y1- $x \mathrm{Cax} \mathrm{Ba} 2-y \mathrm{La} y \mathrm{Cu} 3 \mathrm{O} z$ system from underdoped to overdoped. New Journal of Physics 2009, 11, 113036, DOI: 10.1088/1367-2630/11/11/113036.

21. Hao S J; Jin W T; Guo C Q; Zhang H. Structural and superconducting properties of co-doped $\mathrm{YBa}_{2-\mathrm{xLa}} \mathrm{Cu}_{3-\mathrm{x}} \mathrm{M}_{x} \mathrm{O}_{z}$ and $\mathrm{La}-$ free $\mathrm{YBa}_{2} \mathrm{Cu}_{3}-\mathrm{M} \mathrm{M}_{\mathrm{x}} \mathrm{O}_{z}(\mathrm{M}=\mathrm{Al}, \mathrm{Zn})$ high-TC superconductors. Physica C 2012, 475, 28, DOI: 10.1016/j.physc.2012.01.013.

22. Ambrosch-Draxl C; Auer H; Kouba R; Sherman E Ya; Knoll P; Mayer M. Raman scattering in $\mathrm{YBa}_{2} \mathrm{Cu}_{3} \mathrm{O}_{7}$ : A comprehensive theoretical study in comparison with experiments. Phys. Rev. B 2002, 65, 064501, DOI:10.1103/PhysRevB.65.064501. 